\title{
Manipulating the Lewis antigen specificity of the cholesterol-dependent cytolysin lectinolysin
}

\section{Sara L. Lawrence ${ }^{1}$, Susanne C. Feil' ${ }^{1}$, Jessica K. Holien ${ }^{1}$, Michael J. Kuiper ${ }^{2}$, Larissa Doughty', Olan Dolezal ${ }^{3}$, Terrence D. Mulhern ${ }^{4}$, Rodney K. Tweten ${ }^{5}$ and Michael W. Parker ${ }^{1,4}$ *}

\author{
Biota Structural Biology Laboratory and Australian Cancer Research Foundation Rational Drug Discovery Centre, St. Vincent's Institute of Medical Research, \\ Fitzroy, VIC, Australia \\ ${ }^{2}$ Victorian Life Sciences Computation Initiative, The University of Melbourne, Parkville, VIC, Australia \\ ${ }^{3}$ CSIRO Materials Science and Engineering, Parkville, VIC, Australia \\ ${ }^{4}$ Department of Biochemistry and Molecular Biology, Bio21 Molecular Science and Biotechnology Institute, The University of Melbourne, Parkville, VIC, Australia \\ ${ }^{5}$ Department of Microbiology and Immunology, University of Oklahoma Health Sciences Center, Oklahoma City, OK, USA
}

Edited by:

Paul A. Ramsland, Burnet Institute, Australia

\section{Reviewed by:}

Brian V. Geisbrecht, University of Missouri-Kansas City, USA

Elizabeth Yuriev, Monash University, Australia

*Correspondence:

Michael W. Parker, Biota Structural Biology Laboratory and ACRF Rational Drug Discovery Centre, St. Vincent's Institute of Medical Research, 9 Princes Street, Fitzroy,

VIC 3065, Australia.

e-mail:mparker@svi.edu.au
The cholesterol-dependent cytolysins (CDCs) attack cells by punching large holes in their membranes. Lectinolysin from Streptococcus mitis is unique among CDCs due to the presence of an $\mathrm{N}$-terminal lectin domain that enhances the pore-forming activity of the toxin. We recently determined the crystal structures of the lectin domain in complex with various glycans. These structures revealed the molecular basis for the Lewis antigen specificity of the toxin. Based on this information we have used in silico molecular modeling to design a mutant toxin, which we predicted would increase its specificity for Lewis $y$, an antigen found on the surface of cancer cells. Surprisingly, we found by surface plasmon resonance binding experiments that the resultant mutant lectin domain exhibited higher specificity for Lewis $b$ antigens instead. We then undertook comparative crystallographic and molecular dynamics simulation studies of the wild-type and mutant lectin domains to understand the molecular basis for the disparity between the theoretical and experimental results. The crystallographic results revealed that the net number of interactions between Lewis y and wild-type versus mutant was unchanged whereas there was a loss of a hydrogen bond between mutant and Lewis b compared to wild-type. In contrast, the molecular dynamics studies revealed that the Lewis $b$ antigen spent more time in the binding pocket of the mutant compared to wild-type and the reverse was true for Lewis $y$. The results of these simulation studies are consistent with the conclusions drawn from the surface plasmon resonance studies. This work is part of a program to engineer lectinolysin so that it will target and kill specific cells in human diseases.

Keywords: cholesterol-dependent cytolysins, Lewis antigens, molecular dynamics simulations, protein engineering, surface plasmon resonance, $\mathrm{X}$-ray crystallography

\section{INTRODUCTION}

Lectinolysin (LLY) is a pore-forming toxin derived from some strains of Streptococcus mitis and S. pseudopneumoniae and is a member of the cholesterol-dependent cytolysin (CDC) family (Farrand et al., 2008). These pore-forming bacterial toxins are secreted as soluble monomers that assemble on the surface of cholesterol-rich cell membranes. The resultant pores are composed in excess of 30 monomers, and are greater than $150 \AA$ in diameter (Hotze and Tweten, 2012). Perforation of the membrane by these pores results in cell lysis. The sequence similarity is high (40-80\%) between CDC family members.

Streptococcus mitis can cause diseases such as infective endocarditis and septicemia (Hall and Baddour, 2002; Huang et al., 2002; Gowda etal., 2003; Kennedy etal., 2004). Serum isolates from Kawasaki disease patients were found to contain $S$. mitis human platelet aggregation factor (PAF or Sm-hPAF), so named because it was found to affect light scattering properties of human platelets, thought to be as a consequence of platelet aggregation (Ohkuni etal., 1997). Farrand etal. (2008) found that the Sm-hPAF gene sequence encodes a predicted CDC structure (Rossjohn et al., 1997; Polekhina et al., 2005), most closely related to intermedilysin (Farrand et al., 2008). Having established that PAF was in fact a member of the CDC family, it was renamed lectinolysin, because of its ability to bind carbohydrate (see below).

While LLY shares a number of characteristics typical of CDCs, it has a unique 162 amino acid N-terminal domain (LLYlec; Farrand et al., 2008). Sequence comparisons show that the LLYlec domain shares significant identity with fucose-binding proteins. Glycan array experiments revealed that LLY was highly selective for the difucosylated glycans Lewis y $\left(\mathrm{Le}^{\mathrm{y}}\right)$ and Lewis $\mathrm{b}\left(\mathrm{Le}^{\mathrm{b}}\right)$ antigens (Farrand et al., 2008). Small-angle X-ray scattering (SAXS) modeling predicts that LLY ${ }^{\text {lec }}$ is joined to CDC domain 1 so that the Le antigen-binding site is exposed on the outer surface of the pore (Feil et al., 2012).

Lewis antigens are blood group determinants with very rigid structures (Yuriev et al., 2005). Le ${ }^{\mathrm{b}}$ is a type 1 antigen and adsorbed onto the surface of blood cells. $\mathrm{Le}^{\mathrm{y}}$ is a type 2 antigen which, in 
healthy individuals, is expressed at low levels in tissues including epithelial cells and serum (Yuriev et al., 2005; Westwood et al., 2009). However, expression is elevated on the surface of a wide range of epithelial tumor cells including colon, lung, and ovary (Sakamoto et al., 1986; Miyake et al., 1992; Yin et al., 1996). Thus, $\mathrm{Le}^{\mathrm{y}}$ is a distinctive tumor marker and therefore a promising target for directing drugs to cancer cells.

Various approaches have been taken to produce $\mathrm{Le}^{\mathrm{y}}$-specific cancer therapeutics, including the development of $\mathrm{Le}^{\mathrm{y}}$-specific humanized monoclonal antibodies (Kitamura et al., 1994; Clarke et al., 2000a,b; Scott et al., 2000), and anti Le ${ }^{\mathrm{y}} \mathrm{T}$ cells (Westwood et al., 2005). A chimeric approach has also been taken whereby a $\mathrm{Le}^{\mathrm{y}}$-specific monoclonal antibody was conjugated to the CDC, listeriolysin O, to form an immunotoxin (Bergelt et al., 2009). LLY is a naturally occurring, potential oncotoxin, with in-built tumor targeting specificity for the $\mathrm{Le}^{\mathrm{y}}$ antigen. However, cross reactivity with $\mathrm{Le}^{\mathrm{b}}$ antigen decreases its usefulness as a specific, cancer-targeting molecule.

Previously we have determined the crystal structure of LLYlec, and its complexes with fucose and $\mathrm{Le}^{\mathrm{b}}$ and $\mathrm{Le}^{\mathrm{y}}$ antigens (Feil et al., 2012). The overall fold of the LLYlec domain exhibited similarities with fucolectin domains of the CBM family 98 glycoside hydrolase of Streptococcus pneumonia and Anguilla anguilla agglutinin. The binding of $\mathrm{Le}^{\mathrm{y}}$ and $\mathrm{Le}^{\mathrm{b}}$ to LLYlec was very similar with the exception of an additional hydrogen bond between $\mathrm{Le}^{\mathrm{b}}$ and amino acid residue Tyr62 (Feil et al., 2012).

Using these structures, we have explored the possibility of mutating LLYlec to develop a Le ${ }^{\mathrm{y}}$-specific binding domain. In the work presented here a $\mathrm{Y} 62 \mathrm{H}$ mutation was designed and constructed with the aim of removing the hydrogen bond between the $N$-acetylglucosamine (GlcNAc) of Le ${ }^{\mathrm{b}}$ and LLYlec and instead, gain a hydrogen bond with $\mathrm{Le}^{\mathrm{y}}$. However, binding studies described here show that LLY ${ }^{l e c} Y 62 \mathrm{H}$ has a greater affinity for $\mathrm{Le}^{\mathrm{b}}$ over $\mathrm{Le}^{\mathrm{y}}$, whereas $\mathrm{LLY}^{\mathrm{lec}}$ wt has a preference for $\mathrm{Le}^{\mathrm{y}}$ over $\mathrm{Le}^{\mathrm{b}}$. In order to explore the molecular basis for this unexpected finding we determined the crystal structure of LLY ${ }^{\text {lec }} Y 62 \mathrm{H}$ in complex with $\mathrm{Le}^{\mathrm{b}}$ and $\mathrm{Le}^{\mathrm{y}}$. We then compared these structures with those of the wild-type lectin domain complexed with the same Lewis antigens. The crystallographic studies were complemented by molecular dynamics simulations in order to explore the importance of protein motion and interaction with the ligands over time.

\section{MATERIALS AND METHODS IN SILICO DESIGN OF MUTANTS}

The crystal structures of the lectin domain of LLY in complex with Le ${ }^{b}$ antigen (PDB code: 3 LEK) and $\mathrm{Le}^{\mathrm{y}}$ antigen (PDB code: 3LEG) were visually inspected using $\mathrm{Pymol}^{1}$. In silico mutations were created and assessed also using the Pymol program. Figures were created in Pymol and using Chemaxon software ${ }^{2}$.

\section{SURFACE PLASMON RESONANCE BIOSENSOR BINDING ANALYSIS}

All surface plasmon resonance (SPR) experiments were performed at $25^{\circ} \mathrm{C}$ using a Biacore T200 instrument (GE Healthcare). Direct

\footnotetext{
${ }^{1}$ http://www.pymol.org/
}

${ }^{2}$ http://chemaxon.com/ assays were performed with $\mathrm{Le}^{\mathrm{y}}$ and $\mathrm{Le}^{\mathrm{b}}$ antigens (Sigma) injected over mutant and wild-type LLYlec domain proteins immobilized on a CM5 chip (GE Healthcare). Immobilizations were performed in $1 \times$ HBS-P running buffer [ $10 \mathrm{mM}$ HEPES, $150 \mathrm{mM} \mathrm{NaCl}$, $0.005 \%(\mathrm{w} / \mathrm{v})$ Tween 20]. Binding experiments were performed in $1 \times$ HBS-P + running buffer [10 mM HEPES, $150 \mathrm{mM} \mathrm{NaCl}$, $0.05 \%(w / v)$ Tween 20] containing $1 \mathrm{mM} \mathrm{MgCl}_{2}, 1 \mathrm{mM} \mathrm{CaCl}_{2}$, and $0.2 \mathrm{mg} / \mathrm{ml}$ bovine serum albumin.

$\mathrm{LLY}^{\mathrm{lec}} \mathrm{wt}$ and LLY ${ }^{\mathrm{lec}} \mathrm{Y} 62 \mathrm{H}$ proteins were immobilized in two separate channels on a CM5 chip using a standard amine coupling protocol. Briefly, the chip surface was activated with a single 5 min injection of freshly prepared 1:1 $50 \mathrm{mM} \mathrm{N}$ hydroxysuccinimide:200 mM 3-(N,N-dimethylamino)propyl- $N$ ethylcarbodiimide. Protein coupling was achieved by three $5 \mathrm{~min}$ injections of LLY ${ }^{\text {lec }}$ wt or LLY ${ }^{\text {lec }}$ Y62 $\mathrm{H}$ solution $(50 \mu \mathrm{g} / \mathrm{ml}$ in $10 \mathrm{mM}$ sodium acetate, $\mathrm{pH}$ 5.0). To deactivate residual reactive sites, lectin domain coupling was followed by a 5 min injection of 1 $\mathrm{M}$ ethanolamine ( $\mathrm{pH}$ 8.5). Approximately 3000 response units $\left(\mathrm{RU} ; 1 \mathrm{RU}=1 \mathrm{pg}\right.$ of protein $/ \mathrm{mm}^{2}$ ) of $\mathrm{LLY}^{\mathrm{lec}} \mathrm{wt}$ (channel 2) and 4000 RU of LLY ${ }^{\text {lec }}$ Y62H (channel 3) were coupled. Channel 1 was activated and blocked, as above, for use as a reference surface.

$\mathrm{Le}^{\mathrm{b}}$ and $\mathrm{Le}^{\mathrm{y}}$ were injected, in duplicate, in a twofold dilution series from $1 \mathrm{mM}$ to $7.5 \mu \mathrm{M}$ over the immobilized wild-type and mutant lectin domains. Thirty-second injections of Le antigen at $40 \mu \mathrm{l} / \mathrm{min}$ were followed by a 60 -s dissociation period. Baseline returned to 0 almost immediately after the association phase ended, so regeneration of the surface was not required.

Binding data were processed and analyzed using Scrubber 2 software $^{3}$ (version 2.0c). Rapid association and dissociation rates made data fitting to a kinetic model and subsequent calculation of kinetic rate constants $k_{\mathrm{a}}$ and $k_{\mathrm{d}}$ impractical. Consequently, equilibrium dissociation constants $\left(K_{\mathrm{D}}\right)$ were derived by fitting binding responses at equilibrium to a 1:1 steady-state affinity model available within Scrubber.

\section{MUTAGENESIS, EXPRESSION, AND PURIFICATION OF THE LLYlec DOMAINS}

A mutant of LLY ${ }^{\text {lec }}$, LLY ${ }^{\text {lec }}$ Q190C, with an N-terminal $6 \times$ His tag and a TEV protease cleavage site for His-tag removal, was used in this study. The LLY ${ }^{\text {lec }} \mathrm{Q} 190 \mathrm{C}$ coding region corresponds to LLY residues 38-190 (GenBank accession number AB051299.1). The C-terminal residue was mutated to cysteine (Q190C) for protein labeling studies. The point mutant displays wild-type activity and is located far from the Lewis antigen-binding site (Farrand et al., 2008). Henceforth, LLY ${ }^{\text {lec }}$ Q190C will be described as LLY ${ }^{l e c}$ wt in this work. Expression was carried out in E. coli BL21, and the protein purified using nickel resin and size exclusion chromatography as previously described (Feil et al., 2012). LLY $^{\text {lec }}$ wt was mutated with QuikChange ${ }^{\mathrm{TM}}$ Site-Directed Mutagenesis Kit (Stratagene) to create the Lewis antigen-binding site mutant LLYlecY62H. LLY ${ }^{l e c}$ wt and LLYlec Y62H were purified to $>95 \%$ purity, as determined by SDS gel electrophoresis. Proteins were concentrated to $10 \mathrm{mg} / \mathrm{ml}$ in $10 \mathrm{mM}$ Tris- $\mathrm{HCl} \mathrm{pH}$ 7.2, $10 \mathrm{mM} \mathrm{NaCl}$, and $5 \mathrm{mM}$ dithiothreitol, and were stored at $-80^{\circ} \mathrm{C}$.

${ }^{3}$ www.biologic.com.au 


\section{CRYSTALLIZATION OF THE LLYlec Y62H MUTANT}

All crystallizations were performed using the hanging drop vapor diffusion method at $21^{\circ} \mathrm{C}$. $2 \mu \mathrm{l}$ of protein was mixed with equal volume of precipitant and hung over $0.5 \mathrm{ml}$ of well solution. LLY ${ }^{\mathrm{lec}} \mathrm{Y} 62 \mathrm{H}$ crystallization conditions were determined by fine screening around the conditions that proved successful for the wild-type protein (Feil et al., 2012): $2 \mathrm{M} \mathrm{MgSO}_{4}$ and $100 \mathrm{mM}$ Tris- $\mathrm{HCl}$ buffer $\mathrm{pH}$ 8.2-9.0. Optimization included the addition of the Hampton additive screen (Hampton Research, CA, USA) $10 \%(\mathrm{v} / \mathrm{v})$ in the hanging drops. The optimal crystallization conditions were $2.4 \mathrm{M} \mathrm{MgSO}_{4}$ and $100 \mathrm{mM} \mathrm{KCl}$ with $100 \mathrm{mM}$ Tris-HCl $\mathrm{pH}$ 8.75-9.0. $\mathrm{Le}^{\mathrm{y}}$ and $\mathrm{Le}^{\mathrm{b}}$ antigens were soaked into $\mathrm{LLY}^{\mathrm{lec}} \mathrm{Y} 62 \mathrm{H}$ crystals by adding $1 \mu \mathrm{l} 1 \mathrm{M} \mathrm{MgSO}_{4}, 100 \mathrm{mM}$ Tris- $\mathrm{HCl} \mathrm{pH}$ 9.0, $100 \mathrm{mM} \mathrm{KCl}, 5 \mathrm{mM} \mathrm{Le}^{\mathrm{y}}$ or $\mathrm{Le}^{\mathrm{b}}$ to the crystal drop for $1 \mathrm{~h}$ at $21^{\circ} \mathrm{C}$. Soaks were carried out immediately prior to cryoprotection and flash freezing in liquid nitrogen. Crystals were cryoprotected for $\mathrm{X}$-ray data collection by adding glycerol in increments of $5 \%(\mathrm{v} / \mathrm{v})$ to a final concentration of $20 \%(\mathrm{v} / \mathrm{v})$ to the crystal drops.

\section{CRYSTALLOGRAPHIC STUDIES OF THE LLYlec Y62H MUTANT}

Diffraction data were collected at the MX2 beamline at the Australian Synchrotron in Clayton, Victoria. The data collection was controlled using Blue-Ice software (McPhillips et al., 2002). The diffraction data were processed using the HKL2000 suite (Otwinowski and Minor, 1997). Model building was performed with COOT (Emsley and Cowtan, 2004) using the published wild-type crystal structure of LLYlec (PDB code: 1LE0) as a starting model. Data were refined with REFMAC 5 (Murshudov etal., 1997) from the CCP4 program suite (CCP4, 1994). Restrained positional and isotropic temperature factor refinement was employed until convergence and the refinement was monitored using the $R_{\text {free }}$ residual. The Lewis antigens were fully occupied in the binding sites of both structures and their temperature factors were very similar to those of the surrounding side-chains.

\section{MOLECULAR MODELING OF THE LLYlec INTERACTION WITH THE Le ${ }^{\text {b }}$ AND Le ${ }^{y}$ ANTIGENS}

To investigate the mechanism of association and disassociation between LLYlec and Lewis antigens four molecular models were used: two being the published crystal structures of LLYlec wt bound to either $\mathrm{Le}^{\mathrm{b}}$ or Le $\mathrm{y}^{\mathrm{y}}$ antigen (Feil et al., 2012) and the other two being the crystal structures of LLYlec Y62H, complexed to the same ligands, that are described here. The molecular dynamics program NAMD (Phillips et al., 2005) was used to model ligand disassociation of each complex with a 30 -ns simulation from the initial starting bound conformation, repeated 100 times for a cumulative simulation of $3 \mu \mathrm{s}$ for each system. Models were initially solvated with TIP3 water under periodic boundary conditions of dimensions $48 \AA \times 48 \AA \times 64 \AA$. Charges were neutralized with $\mathrm{NaCl}$ for a total ionic concentration of $150 \mathrm{mM}$. The bound calcium ion seen in the crystal structures was included in the simulations. Dynamic molecular modeling was conducted at a theoretical $\mathrm{pH}$ of 7.4. Each simulation run started with an equilibration phase of $0.5 \mathrm{~ns}$, where the protein backbone and Lewis antigen was harmonically constrained to their starting positions at $310 \mathrm{~K}$ and an NPT ensemble (an isothermal and isobaric ensemble where the number of moles $(\mathrm{N})$, pressure $(\mathrm{P})$, and temperature $(\mathrm{T})$ are conserved). Subsequent 30-ns production simulations were run with no constraints at $310 \mathrm{~K}$ under NVT ensembles [a canonical ensemble where the number of moles $(\mathrm{N})$, volume $(\mathrm{V})$, and temperature $(\mathrm{T})$ are conserved]. Trajectory snapshots were captured every 100 ps. At the completion of 100 repeat simulations, all production runs per model were consolidated into a single trajectory and the frames root-mean-square (rms) deviation were centered, based on the LLY alpha carbon protein backbone. The Lewis antigen was then subject to clustering analysis, whereby the top 20 ligand conformational clusters with a cut-off of $1.5 \AA$ were generated and averaged. Ligand clusters with a rms deviation value less than $3.5 \AA$ from the initial position were considered to be in a bound conformation.

\section{RESULTS}

\section{IN SILICO DESIGN OF MUTANTS}

The difference between $\mathrm{Le}^{\mathrm{y}}$ and $\mathrm{Le}^{\mathrm{b}}$ antigens is the core disaccharide linkage ( $\mathrm{Gal} \beta 1-4$ versus $\mathrm{Gal} \beta 1-3$ ) with an opposing projection of the $N$-acetyl glucosamine (GlcNAc) $N$-acetyl and $-\mathrm{CH}_{2} \mathrm{OH}$ groups of the $\mathrm{N}$-acetylglucosamine monosaccharide as shown in Figure 1. The overall Lewis determinants maintain the same conformation in both free and bound states.

In the crystal structure of LLYlec wt the GlcNAc of Le ${ }^{\mathrm{b}}$ is within hydrogen bonding distance (3.3 $\AA$ ) of Tyr62 (Figure 2A; Feil et al., 2012). This interaction is not able to occur with $\mathrm{Le}^{\mathrm{y}}$, thus suggesting that mutating Tyr62 may alter the affinity for Le $\mathrm{L}^{\mathrm{b}}$. Various mutations of this residue were created in silico. Mutation to a histidine residue would maintain structural similarity of the binding site whilst also creating the potential for a new interaction between the histidine side-chain and $\mathrm{Le}^{\mathrm{y}}$ as illustrated in Figure 2B. Replacement of Tyr62 with His was expected to remove the interaction between $\mathrm{Le}^{\mathrm{b}}$ and the hydroxyl group of Tyr62 (Figure 2A).

\section{SPR BIOSENSOR BINDING ANALYSIS}

Purified LLY ${ }^{\text {lec }} \mathrm{Y} 62 \mathrm{H}$ yield was $20 \mathrm{mg} / \mathrm{l}$ E. coli BL21 culture in Luria-Bertani (LB) broth. This yield was equivalent to that found for $L_{L Y}{ }^{l e c} w t$. The sensorgrams from SPR experiments between the Lewis antigens $\left(\mathrm{Le}^{\mathrm{b}}\right.$ and $\mathrm{Le}^{\mathrm{y}}$ ) and either wt or $\mathrm{LLY}^{\mathrm{lec}} \mathrm{Y} 62 \mathrm{H}$ are shown in Figure 3. The estimated $K_{\mathrm{D}}$ values are listed in Table 1. The data indicate low affinity binding interactions of the Lewis antigens with the lectin domains. The wild-type lectin domain (LLY $\left.{ }^{l e c} w t\right)$ has more than threefold greater affinity for $\mathrm{Le}^{\mathrm{y}}$ over $\mathrm{Le}^{\mathrm{b}}$. The LLY ${ }^{\mathrm{lec}} \mathrm{Y} 62 \mathrm{H}$ mutant has a 1.6-fold greater preference for $\mathrm{Le}^{\mathrm{b}}$ over $\mathrm{Le}^{\mathrm{y}}$. The mutation causes an overall 2.5-fold decrease in the lectin domain's affinity for $\mathrm{Le}^{\mathrm{y}}$ and doubles its affinity for $\mathrm{Le}^{\mathrm{b}}$ over the wild-type domain. Overall, the data show that wild-type LLY ${ }^{\text {lec }}$ preferentially binds Le ${ }^{y}$ antigen while the mutant LLY $^{\text {lec } Y 62 H ~ e x h i b i t s ~ h i g h e r ~ a f f i n i t y ~ f o r ~ L e ~}{ }^{b}$ than for $\mathrm{Le}^{\mathrm{y}}$.

\section{CRYSTAL STRUCTURES OF LLYlec Y62H IN COMPLEX WITH Le ${ }^{\mathrm{y}}$ AND Le $\mathrm{e}^{\mathrm{b}}$ ANTIGENS}

LLY ${ }^{\text {lec }}$ Y62 $\mathrm{H}$ crystallization was optimized in $100 \mathrm{mM}$ Tris $\mathrm{pH}$ 8.75-9.0, 2.4 $\mathrm{M} \mathrm{MgSO}_{4}$, and $100 \mathrm{mM} \mathrm{KCl}$. Small bipyramidal crystals of dimensions $0.15 \mathrm{~mm} \times 0.12 \mathrm{~mm} \times 0.12 \mathrm{~mm}$ appeared in 6-8 weeks. After 8 months some crystals had grown 


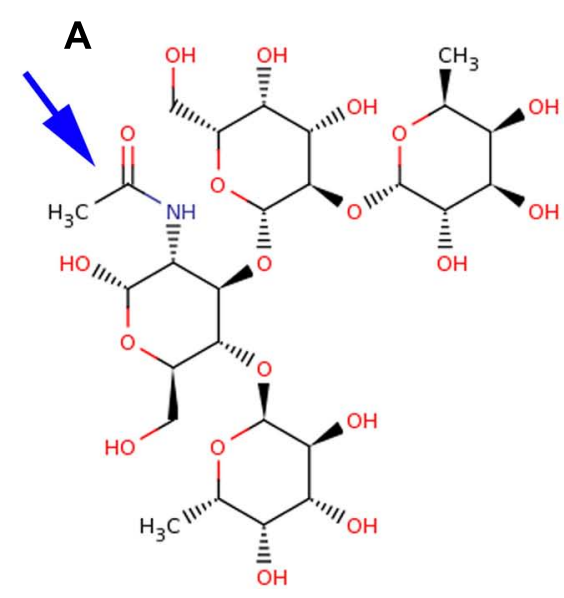

FIGURE 1 | Structural comparison of $\mathrm{Le}^{\mathrm{b}}$ and $\mathrm{Le}^{\mathrm{y}}$ antigens. Lewis antigens $L^{b}{ }^{b}(\mathbf{A})$ and $L^{y}(\mathbf{B})$ are structurally very similar. They differ in the

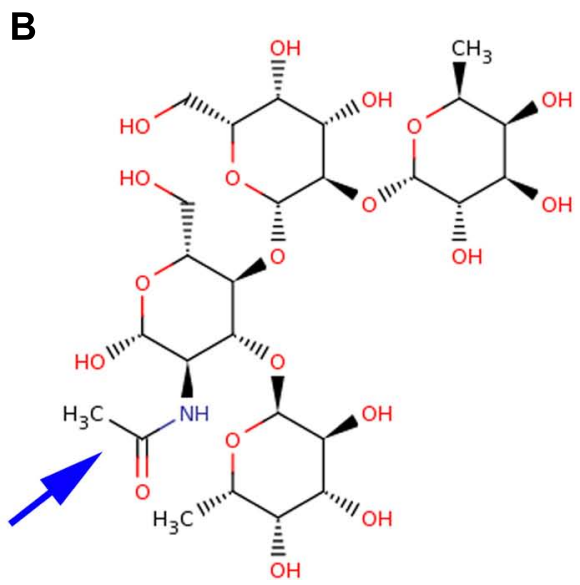

core disaccharide linkages and the opposing projections of their $N$-acetyl (blue arrow) and $\mathrm{CH}_{2} \mathrm{OH}$ groups of the GlcNAc moiety.

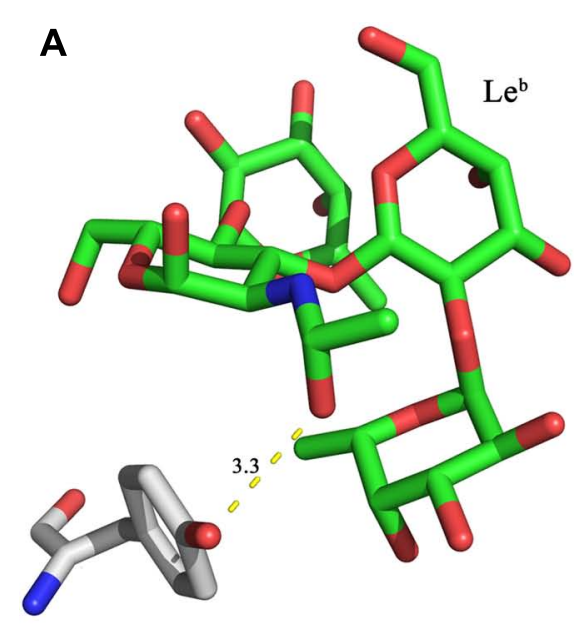

Tyr62

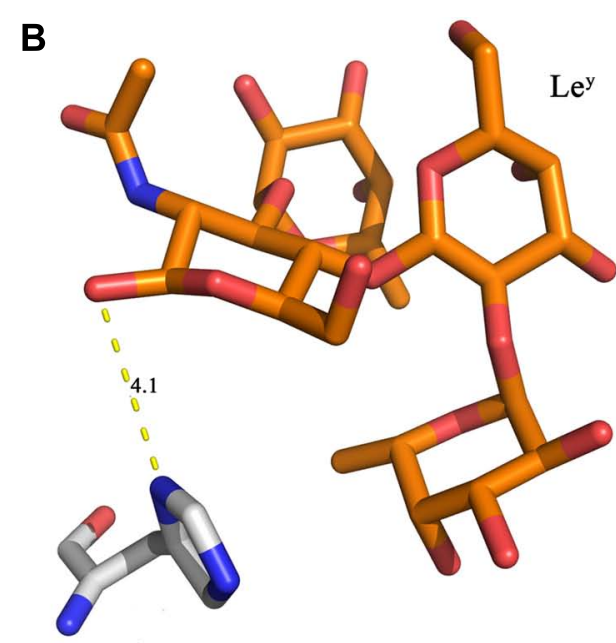

His62

FIGURE 2 | In silico LLYlecY62H mutant design. (A) Le ${ }^{b}$ is within hydrogen bonding distance (3.3 $\AA$ ) of Tyr62 from the LLYlec wt domain. (B) A Y62H mutation was designed to retain similar structural properties to tyrosine, with the possibility that it may also result in a hydrogen bond to the Le ${ }^{Y}$ antigen. Yellow dashed lines denote hydrogen bonds.

to $0.25 \mathrm{~mm} \times 0.2 \mathrm{~mm} \times 0.2 \mathrm{~mm}$. In contrast, LLY ${ }^{\mathrm{lec}}$ wt crystals appeared after 5 days in $100 \mathrm{mM}$ Tris $\mathrm{pH} 8.2-8.4,2.0 \mathrm{M}$ $\mathrm{MgSO}_{4}$ with the same morphology as LLYlecY62H, but reach the same size within 10 days (Feil et al., 2012). Lewis antigens were soaked into crystals immediately prior to cryoprotection and freezing.

The crystal structures of the LLY ${ }^{\text {lec }} Y 62 \mathrm{H}$ in complex with the Lewis antigens was performed as described in Materials and Methods. The crystal structures have been deposited in the PDB with codes: 4GWJ and 4GWI for the Lewis b and Lewis y complexes, respectively. Data statistics for the structures are listed in Table 2.

LLY ${ }^{\text {lec }}$ adopts an eight-stranded $\beta$-sandwich fold, composed of a five-stranded anti-parallel $\beta$-sheet on one side and a three-stranded anti-parallel $\beta$-sheet on the other side. Three short $\alpha$-helices separate $\beta$-strands 1 and 2 and 3 and 4 . There is one calcium ion in the structure that is in the same position as observed in structurally related fucolectin domains. There is also a metal ion that is covalently bound to His80, as well as five water molecules, all arranged in octahedral geometry. In our previous work we identified the ion as either a $\mathrm{Ni}^{2+}$ or $\mathrm{Mg}^{2+}$ ion (Feil et al., 2012) and here we have chosen the latter possibility. The Le $\mathrm{y}^{\mathrm{y}}$ and Le $\mathrm{b}^{\mathrm{b}}$ antigenbinding site is in a cleft at one end of the molecule. A more detailed description of the structure has been reported elsewhere (Feil et al., 2012). In the new mutant structures some additional N-terminal residues are observed (three extra in the $\mathrm{Le}^{\mathrm{y}}$ structure and one extra in the Le $\mathrm{e}^{\mathrm{b}}$ structure), compared to the published wild-type structures (see Table 2).

The structures of the $\mathrm{LLY}^{\mathrm{lec}}{ }_{\mathrm{wt}}-\mathrm{Le}^{\mathrm{y}}$ (Figure 4A) and LLY $^{\text {lec }}$ Y62H-Le ${ }^{\mathrm{y}}$ (Figure 4B) superimpose very closely, with a 


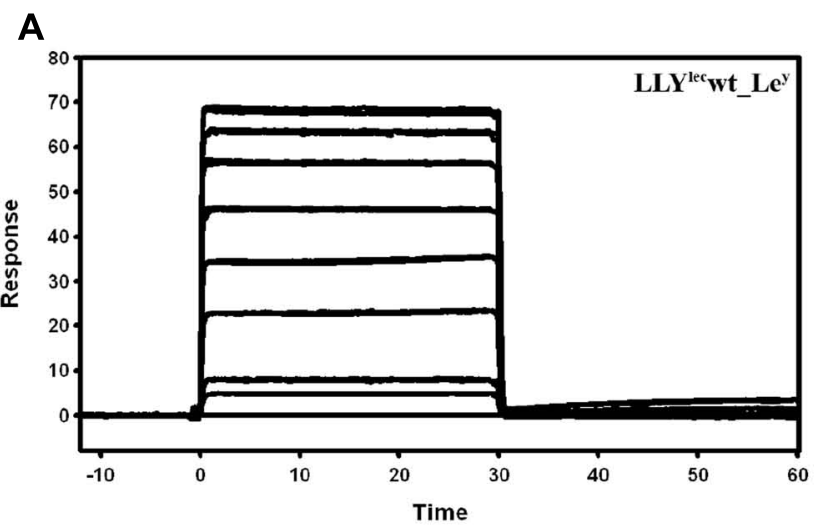

C

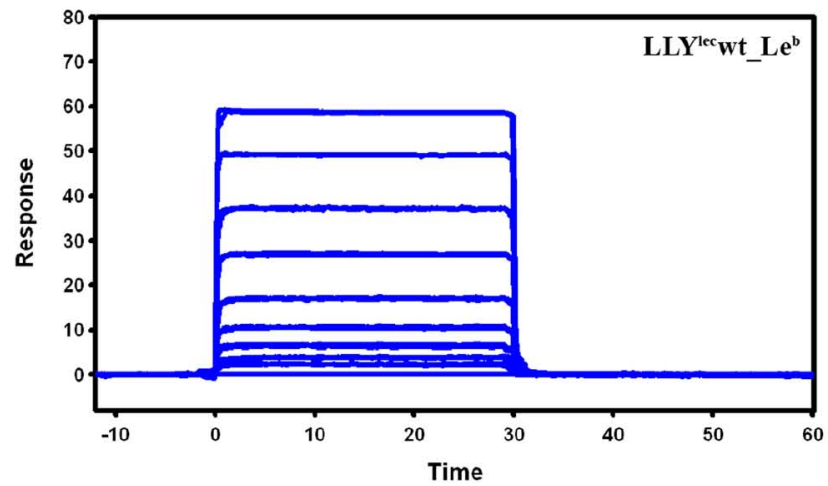

E

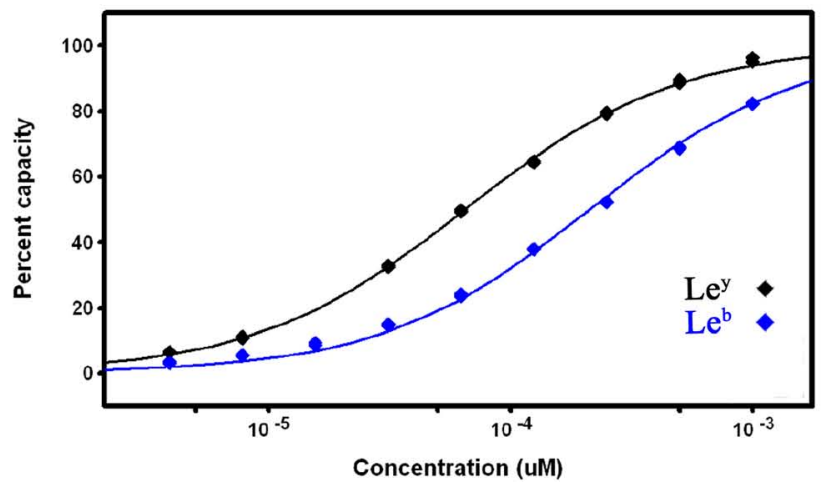

FIGURE 3 | SPR measurements of immobilized LLYlec $w$ t and LLYlecY62H. $\mathrm{Le}^{\mathrm{Y}}$ antigen and Le $\mathrm{b}^{\mathrm{b}}$ antigen were injected at concentrations of 7.6-1000 $\mu \mathrm{M}$. (A) LLYlec wt vs Ley, (B) LLYlecY62H vs Ley, (C) LLYlec wt vs Le ${ }^{b}$, (D)

rms deviation of the alpha carbon atoms of $0.1 \AA$. A third metal ion binding site is observed in the mutant structure and we have tentatively identified it as a $\mathrm{Mg}^{2+}$ ion binding site due to the very high concentrations of this ion in the crystallization buffer. The $\mathrm{Mg}^{2+}$ ion is bound between the carboxylate of Asp97 and the sidechain carbonyl of Gln54 and four water molecules, all arranged in octahedral geometry around the metal ion. The water structure
B

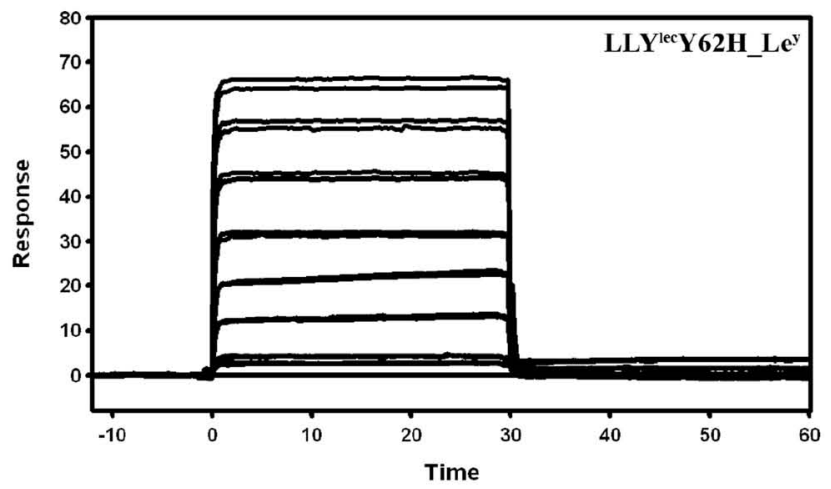

D

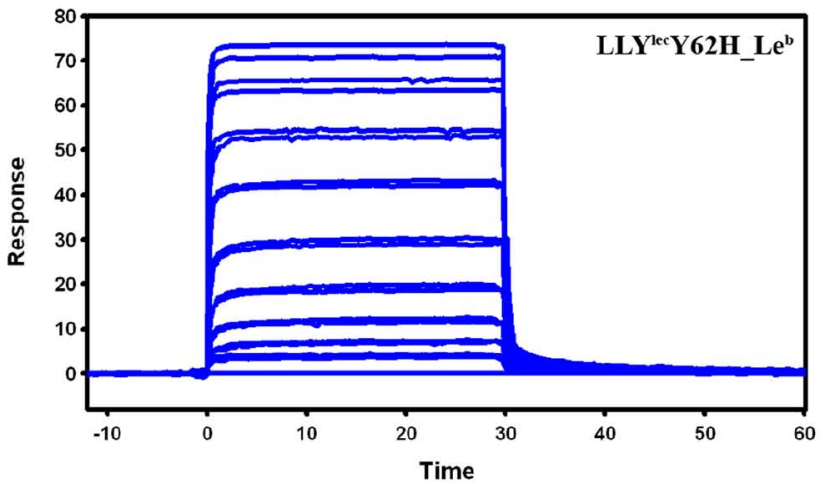

$\mathbf{F}$

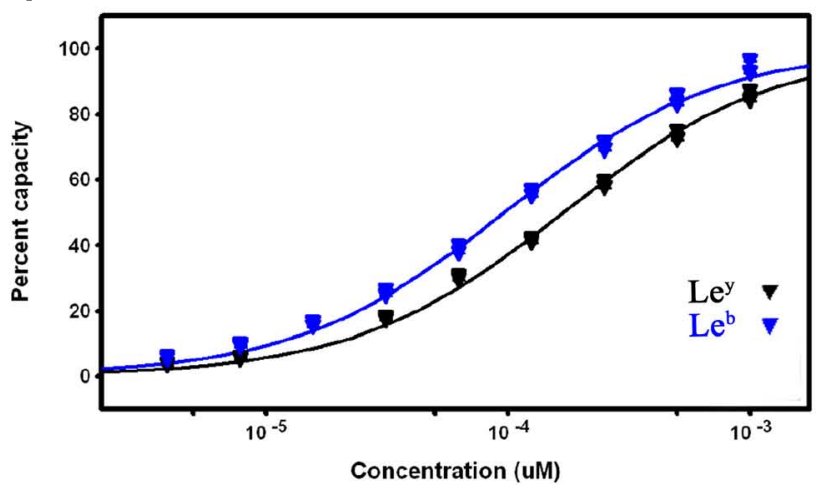

LLYlecY62H vs Le ${ }^{b}$. Responses at equilibrium fitted to simple 1:1 binding isotherms for Ley (black lines) and Le ${ }^{b}$ (blue lines) interacting with (E) $\mathrm{LLYlec}_{W t}$ and (F) LLYlecY62H mutant. Duplicate binding data sets are shown.

is the same as in $\mathrm{LLY}^{\mathrm{lec}} \mathrm{wt}-\mathrm{Le}^{\mathrm{y}}$ apart from a water molecule that is positioned between the hydroxyl group of Tyr62 and fucose 1 (Fuc 1; Figure 4A). This water molecule is shifted by $1.4 \AA$ toward the NE2 of His62 in the mutant structure (Figure 4B), compared to wild-type (Figure 4A), to optimize its interaction with the His side-chain. LLYlecY62H forms the same 19 van der Waals interactions with $\mathrm{Le}^{\mathrm{y}}$ compared to the wild-type protein: 
Table 1 | Estimated equilibrium dissociation constants $\left(K_{D}\right)$ for LLYlecY62H and LLYlec wt domains interacting with $\mathrm{Le}^{\mathrm{y}}$ and $\mathrm{Le}^{\mathrm{b}}$ antigens.

\begin{tabular}{|c|c|c|}
\hline & $\operatorname{LLYlec} w t K_{\mathrm{D}}(\mu \mathrm{M})$ & LLYlecY62H $K_{\mathrm{D}}(\mu \mathrm{M})$ \\
\hline $\operatorname{Le}^{y}$ & $78 \pm 9$ & $202 \pm 31$ \\
\hline $\mathrm{Le}^{\mathrm{b}}$ & $234 \pm 12$ & $127 \pm 14$ \\
\hline
\end{tabular}

15 with Fuc 1, 1 with Gal, 2 with GlcNAc, and 1 with Fuc 2. The B-factor of $\mathrm{Le}^{\mathrm{y}}$ is $53.5 \AA^{2}$ in the mutant (overall B-factor of protein is $29 \AA^{2}$ ), compared to $38.2 \AA^{2}$ in the wild-type protein (overall B-factor of protein complex is $21 \AA^{2}$ ). Overall, there is no net change in the number of potential hydrogen binding and van der Waals interactions between wild-type and mutant $\mathrm{Le}^{\mathrm{y}}$ complexes.

Superposition of the structures of the LLYlec $w t-L e^{b}$ and LLY $^{\text {lec }}$ Y $62 \mathrm{H}-\mathrm{Le}^{\mathrm{b}}$ gives a rms deviation of the alpha carbon atoms of $0.1 \AA$. In addition to the $\mathrm{Ca}^{2+}$ and $\mathrm{Mg}^{2+}$ ion binding sites seen in the wild-type structure there is the additional $\mathrm{Mg}^{2+}$ ion binding site as observed in the LLY ${ }^{l e c} \mathrm{Y} 62 \mathrm{H}-\mathrm{Le}^{\mathrm{y}}$ crystal structure. In the structure of LLY ${ }^{l e c} w t-L e^{b}$ there is one hydrogen bond from the hydroxyl group of Tyr62 to the $N$-acetyl moiety of $\mathrm{Le}^{\mathrm{b}}$ and a second one via a water molecule to the hydroxyl group of Fuc 1 (Figure 4C). In the LLY ${ }^{l e c} Y 62 H-L e^{b}$ structure (Figure 4D) the bond from Tyr62 to the $N$-acetyl is replaced by a bifurcated hydrogen bond from His62 to the hydroxyl group of the GlcNAc ring and the hydroxyl of the $N$-acetyl moiety off the ring. Overall, there was a net loss of a potential hydrogen bond as the watermediated hydrogen bond between Tyr62 and Fuc 1 seen in the LLY $^{l e c}$ wt-Le $e^{b}$ structure has been lost in LLYlec H62-Le ${ }^{b}$. The water molecules between the protein and $\mathrm{Le}^{\mathrm{b}}$ are conserved in wildtype and mutant structures. LLYlec $Y 62 \mathrm{H}$ forms the same 23 van der Waals interactions with $\mathrm{Le}^{\mathrm{b}}$ as does the wild-type protein: 15 with Fuc 1, 1 with Gal, 2 with GlcNAc, and 4 with Fuc 2. The average B-factor of Le $\mathrm{b}^{\mathrm{b}}$ is $43.8 \AA^{2}$ in LLY ${ }^{\mathrm{lec}} \mathrm{Y} 62 \mathrm{H}-\mathrm{Le} \mathrm{b}^{\mathrm{b}}$ (overall $\mathrm{B}$-factor of protein complex is $23 \AA^{2}$ ) which is very similar to $36.7 \AA^{2}$ observed in the wild-type complex (overall B-factor of protein complex is $18 \AA^{2}$ ). Thus, overall, there is a loss of one potential hydrogen bond between $\mathrm{Le}^{\mathrm{b}}$ and mutant compared to wild-type.

Superposition of the crystal structures of LLY ${ }^{l e c} Y 62 H-L e^{b}$ and $\mathrm{LLY}^{\mathrm{lec}} \mathrm{Y} 62 \mathrm{H}-\mathrm{Le}^{\mathrm{y}}$ results in a rms deviation on the alpha carbons atoms of $0.1 \AA$. Fuc 1 and the Gal moieties of the $\mathrm{Le}^{\mathrm{y}}$ and $\mathrm{Le}^{\mathrm{b}}$ antigens superimpose quite well whereas GlcNAc and Fuc 2 are shifted. GlcNAc in $\mathrm{Le}^{\mathrm{b}}$ moves $0.6 \AA$ toward the protein due to a water-mediated hydrogen bond between the oxygen of the GlcNAc- $\mathrm{CH}_{2} \mathrm{OH}$ group to the backbone oxygen of His62. Likewise, Fuc 2 is shifted by about $0.6 \AA$ because the same interaction is not present in the $\mathrm{Le}^{\mathrm{y}}$ complex.

\section{MOLECULAR SIMULATIONS OF LEWIS ANTIGEN INTERACTIONS}

A total of 30,000 trajectory frames $(100 \times 30 \mathrm{~ns})$ were collected for each Lewis antigen in complex with either $\operatorname{LLY}^{\mathrm{lec}} \mathrm{wt}$ or the $\mathrm{LLY}^{\mathrm{lec}} \mathrm{Y} 62 \mathrm{H}$. In the wild-type simulations, the Le $\mathrm{e}^{\mathrm{y}}$ antigen resided within the binding pocket more than the Le $\mathrm{b}^{\mathrm{b}}$ antigen (Table 3 ). However, when Y62 was mutated to a His this trend was reversed,
Table 2 | Crystallographic data processing and refinement statistics for $\mathrm{LLY}^{\mathrm{lec}}$ wt and LLYlecY62H crystal structures in complex with Le ${ }^{\mathrm{Y}}$ and $L^{b}$ antigens.

\begin{tabular}{|c|c|c|}
\hline & LLYlecy62H-Le ${ }^{y}$ & LLYlecY62H-Le ${ }^{b}$ \\
\hline \multicolumn{3}{|l|}{ Data collection } \\
\hline Space group & $P 4_{3} 2_{1} 2$ & $P 4_{3} 2_{1} 2$ \\
\hline Unit cell dimensions $(\AA)$ & $67.1,67.1,99.4$ & $66.9,66.9,99.4$ \\
\hline Wavelength $(\AA ̊)$ & 0.95 & 0.95 \\
\hline Temperature (K) & 100 & 100 \\
\hline Maximum resolution $(\AA ̊)$ & 1.6 & 1.6 \\
\hline No. of observations & 426,761 & 404,032 \\
\hline No. of unique reflections & 30,563 & 29,510 \\
\hline Redundancy & 14.0 & 13.7 \\
\hline Data completeness (\%) & $99.2(93.7)$ & $96.7(79.3)$ \\
\hline $\mid / \sigma_{1}$ & $19.6(7.1)$ & $16.9(5.3)$ \\
\hline$R_{\text {merge }}(\%)^{\mathrm{A}}$ & $11.5(45.8)$ & $10.9(57.1)$ \\
\hline
\end{tabular}

\section{Refinement}

Non-hydrogen atoms

$\begin{array}{lll}\text { Protein } & 1124 & 1103 \\ \text { Water } & 146 & 150 \\ \mathrm{Mg}^{2+} & 2 & 3 \\ \mathrm{Ca}^{2+} & 1 & 1 \\ \text { Ligands } & 46 & 46 \\ \text { Resolution }(\AA) & 1.6 & 1.6 \\ R_{\text {work }}(\%)^{\mathrm{B}} & 16.9 & 18.0 \\ R_{\text {free }}(\%) & 19.1 & 21.4\end{array}$

Rms deviations from ideal geometry

$\begin{array}{lll}\text { Bond lengths }(\AA) & 0.028 & 0.028 \\ \text { Bond angles }(\theta) & 1.4 & 1.4 \\ \text { Bonded } B^{\prime} \text { s } & 3.9 & 4.1 \\ \text { Mean } B\left(\AA^{2}\right) & & \end{array}$

$\begin{array}{lll}\text { Main-chain } & 22.8 & 27.0 \\ \text { Side-chain } & 28.2 & 31.9 \\ \text { Water } & 36.9 & 29.4 \\ \text { Ligand } & 53.5 & 43.8 \\ \text { Residues observed } & 41 \text { to } 184 & 43 \text { to } 184 \\ \text { Residues in most favored regions } & 87.7 & 88.4\end{array}$

of the Ramachandran plot (\%)

Residues in the disallowed regions

0

0

of the Ramachandran plot (\%)

The values in parentheses are for the highest resolution bin lapproximately $0.1 \AA$ width). ${ }^{A} R_{\text {merge }}=\Sigma_{h k l} \Sigma_{i} l_{i}-\left\langle|>|||<|>|\right.$, where $I_{i}$ is the intensity for the ith measurement of a symmetry related reflection with indices $h, k, l .{ }^{B} R_{\text {work. }}=\Sigma|| F_{o b s} l-$ $\left|F_{c a l c}\right||\Sigma| F_{o b s} \mid$, where $F_{o b s}$ and $F_{c a l c}$ are the observed and calculated structure factor amplitudes, respectively.

with $\mathrm{Le}^{\mathrm{b}}$ residing in the binding pocket more than $\mathrm{Le}^{\mathrm{y}}$. These results are reflective of the SPR binding data, in that LLYlec $w$ t has a higher affinity for Le $e^{y}$ over Le ${ }^{b}$, whereas LLY ${ }^{\text {lec }} Y 62 \mathrm{H}$ has a higher affinity for $\mathrm{Le}^{\mathrm{b}}$ over for $\mathrm{Le}^{\mathrm{y}}$. 

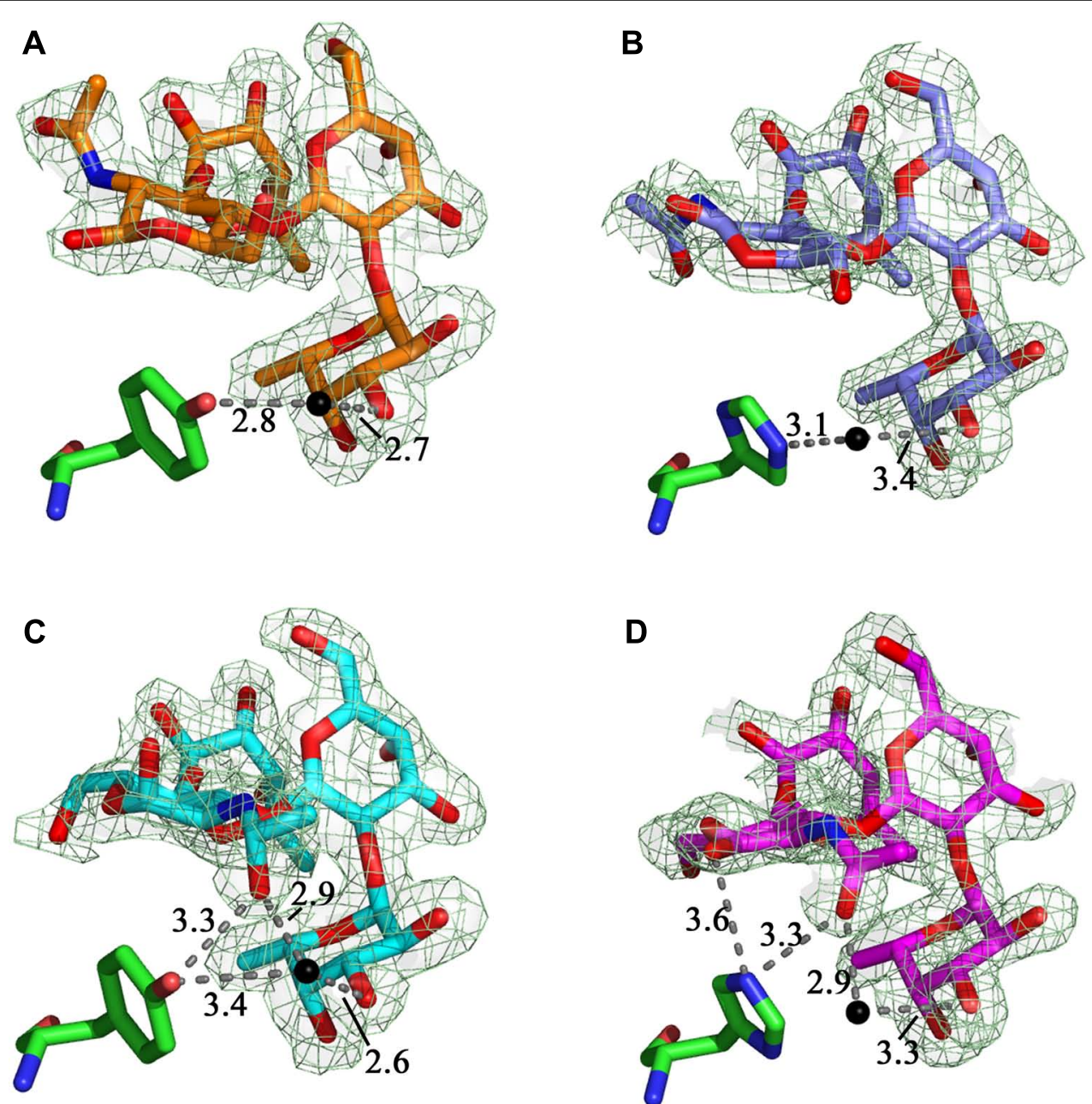

FIGURE 4 | LLYlec $w$ t and LLYlecy62H domains in complex with Lewis antigens. Comparison between (A) the $\mathrm{LLY}^{\mathrm{lec}}{ }_{\mathrm{Wt}}-\mathrm{Le}^{\mathrm{Y}}$ complex (orange carbons, green Tyr62) and (B) the LLYlecY62H-LeY complex (purple carbons, green His62) shows that the water molecule shifts toward the histidine residue in $\mathrm{LLYlec} 62 \mathrm{H}-\mathrm{Le}^{\mathrm{Y}}$. The water-mediated hydrogen bond to the hydroxyl
Lewis antigen dissociation rates were plotted as a function of occupancy (defined as $<3.5 \AA$ deviation from the original binding site) for all 100 simulations (Figure 5). This indicated a rapid dissociation rate which supports the SPR data where the on and off rates were too fast to be calculated. There is

Table 3 | Cumulative totals of bound Lewis antigen over the course of 100 independent, 30 ns simulations starting from the Lewis bound conformation.

\begin{tabular}{lll}
\hline & wt & LLYlecY62H \\
\hline $\mathrm{Le}^{y}$ & $17,260(57.5 \%)$ & $13,835(46.1 \%)$ \\
$\mathrm{Le}^{\mathrm{b}}$ & $14,824(49.4 \%)$ & $16,080(53.6 \%)$ \\
\hline
\end{tabular}

All binding events within $3.5 \AA$ rms deviation of the original antigen bound conformation were considered "bound." an overall trend for the antigens to occupy the binding site less as the simulations progressed. However, it is important to note that the curves are not purely exponential and contain distinct areas of peaks and troughs. This is indicative of the ligands moving rapidly in and out of the binding site of both LLY ${ }^{\mathrm{lec}} \mathrm{wt}$ and LLY ${ }^{\text {lec }}$ $62 \mathrm{H}$.

The simulation data reiterates the SPR finding that mutation of LLY ${ }^{\mathrm{lec}}$ Tyr62 to His62 has changed the binding specificity from a preference for $\mathrm{Le}^{\mathrm{y}}$ by wild-type to a specificity for $\mathrm{Le}^{\mathrm{b}}$ by the LLYlecY62H mutant.

\section{DISCUSSION}

The high level of expression of $\mathrm{Le}^{\mathrm{y}}$ on the surface of epithelial tumor cells and low level expression elsewhere makes cell-bound $\mathrm{Le}^{\mathrm{y}}$ a highly attractive target for anti-tumor agent delivery. In addition, the rigid nature and distinctive structure of $\mathrm{Le}^{\mathrm{y}}$ lends itself to the ready development of highly specific protein recognition 


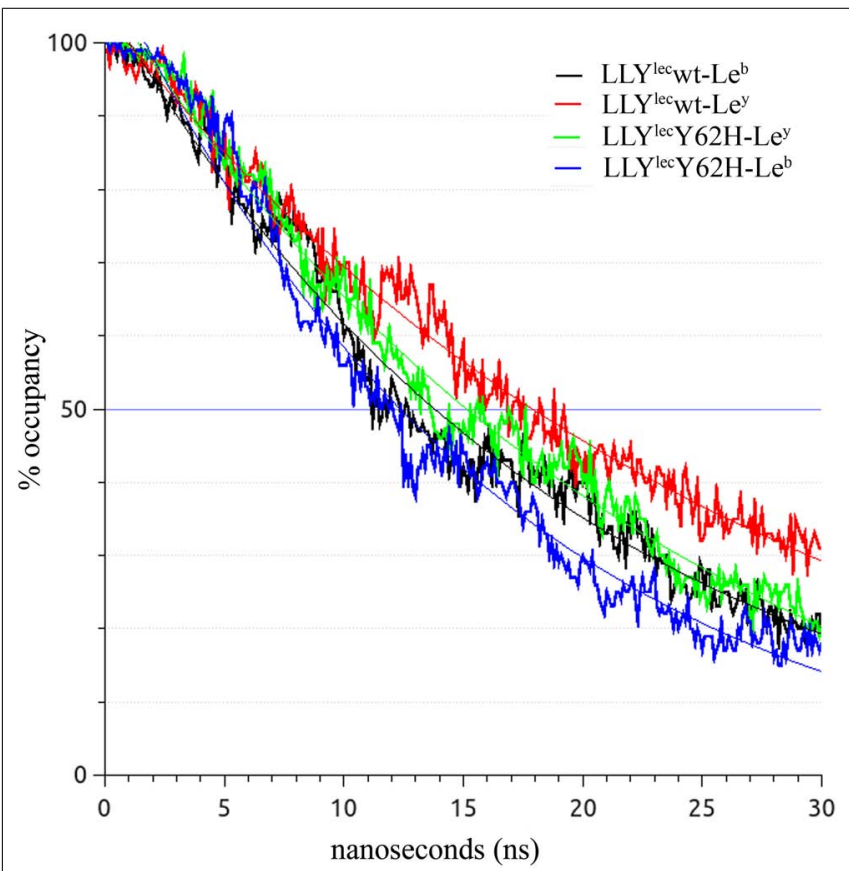

FIGURE 5 | Molecular dynamics modeling of the association of Lewis antigens with the LLYlec binding sites. Traces are averaged for all 100 simulations. Overall, the Lewis antigens occupied the binding site of either the wild-type or LLYlecY62H less as the simulation progressed. However there are numerous areas of re-association, resulting in a spike in occupancy.

domains, whether in antibodies or other glycan-binding proteins.

Previously, we published the crystal structures of LLY ${ }^{l e c}$ wt in complex with the Le ${ }^{\mathrm{b}}$ and $\mathrm{Le}^{\mathrm{y}}$ antigens (Feil et al., 2012). These structures, together with computational modeling, were used to design and construct a glycan-binding domain with altered specificity for the Lewis antigens. The LLYlec $\mathrm{Y} 62 \mathrm{H}$ mutation was suggested due to its structural similarity of His to Tyr and the potential to create a new interaction with $\mathrm{Le}^{\mathrm{y}}$.

Surface plasmon resonance binding studies showed that $L_{L Y}{ }^{l e c}$ wt has a higher affinity for the Le ${ }^{y}$ antigen over Le ${ }^{b}$. This relationship was reversed for the designed LLYlecY62H mutant, i.e., the Le ${ }^{\mathrm{b}}$ antigen had higher affinity than the $\mathrm{Le}^{\mathrm{y}}$ antigen. These results were unexpected, as the molecular modeling predicted that mutation of Tyr62 to His should decrease the number of hydrogen bonds with $\mathrm{Le}^{\mathrm{b}}$ and increase the number of potential hydrogen bonds with $\mathrm{Le}^{\mathrm{y}}$. The expectation was that the mutation would lead to an increase in affinity for $\mathrm{Le}^{\mathrm{y}}$ and decrease in affinity for $\mathrm{Le}^{\mathrm{b}}$, but in practice we found the reverse to be true.

To experimentally determine the interactions formed in the binding site as a result of the LLYlec $\mathrm{Y} 62 \mathrm{H}$ mutation, we solved the crystal structures of LLY ${ }^{\text {lec }} \mathrm{Y} 62 \mathrm{H}$ in complex with the Lewis antigens. In the LLY ${ }^{l e c} Y 62 \mathrm{H}-\mathrm{Le}^{\mathrm{y}}$ complex, there was no additional bond formation between the Lewis antigen and His62, only a shift of a water molecule toward the His, as compared to its position relative to Tyr62 in the wild-type (Figures 4A,B). In the $\mathrm{Le}^{\mathrm{b}}$ complex there was a net loss of a potential hydrogen bond to
His62 (Figures 4C,D). In summary, the crystal structures did not show the bonding as predicted from the in silico mutant model (Figure 2) but revealed a net decrease in hydrogen bonding interactions to $\mathrm{Le}^{\mathrm{b}}$ and no net change in bonding interactions with $\mathrm{Le}^{\mathrm{y}}$. However, the SPR data revealed that the mutant had an increased affinity for $\mathrm{Le}^{\mathrm{b}}$ and a decreased affinity for $\mathrm{Le}^{\mathrm{y}}$.

Crystal structures are a static average snapshot of what happens in solution, so we used molecular dynamics studies to simulate the lectin domain-Lewis antigen binding mechanism in solution. In these simulations it was observed that the amino acid residues around the Lewis antigen binding site are mobile, particularly Tyr62. Thus, the inferred hydrogen bonding interactions between protein and ligands observed in the crystal structures may not necessarily be persistent in solution. This may help explain the unexpected consequences of our mutation.

The molecular dynamics studies provided us with complementary results to the SPR data: both approaches showed that the association and dissociation rates with Lewis antigens are extremely rapid, and that the relative affinities between antigens and wild-type or LLYlecY62H domains are generally weak. The SPR data showed the strongest interaction being that of LLY ${ }^{l e c} w t$ to $\mathrm{Le}^{\mathrm{y}}$ with a $K_{\mathrm{D}}$ of $78 \mu \mathrm{M}$ and the weakest between LLY $^{\mathrm{lec}}{ }_{\mathrm{wt}}$ and $\mathrm{Le}^{\mathrm{b}}$ with a $K_{\mathrm{D}}$ of $234.4 \mu \mathrm{M}$. Relative affinities of the wild-type or LLY ${ }^{\text {lec }} \mathrm{Y} 62 \mathrm{H}$ binding sites for antigens was measured in the simulations by calculating how much time the Lewis antigens spent in the binding pocket. These dynamics studies agreed with the general trend of the biological results, suggesting that there was a reversal of Lewis antigen affinity when Tyr62 was mutated to a His. Interestingly, and again in concurrence with SPR data, the dynamics studies also suggest that the Lewis antigens bind weakly and transiently to the LLY ${ }^{\text {lec }}$ domain.

A recent example of protein engineering to alter Lewis antigen specificity is that of Norovirus virion protein 1 (NoV VP1), which specifically binds Le ${ }^{\mathrm{b}}$ (Kubota et al., 2012). However, NoV VP1 has a deeper binding site with more extensive contacts to the Lewis antigen structure than seen in LLY ${ }^{\text {lec }}$. A single residue (Gln) in the $\mathrm{Le}^{\mathrm{b}}$ binding site was mutated (to Asn) with the aim of increasing the affinity of the protein for $\mathrm{Le}^{\mathrm{b}}$. The mutation increased the width of the binding site, allowing bonding between both fucose rings and amino acid side-chains. As expected, the mutant protein displayed a higher affinity for Le ${ }^{\mathrm{b}}$.

In contrast, designing a high affinity $\mathrm{Le}^{\mathrm{y}}$-specific LLY ${ }^{\mathrm{lec}}$ mutant is more challenging: the crystal structures show that the Lewis antigens sit in a shallow hydrophobic pocket and that the only hydrogen bonds are between the basic residues His85, Arg112, and Arg120, and the $\alpha 1-2$ linked fucose. Few contacts are made between LLY lec residues and other carbohydrate components of the $\mathrm{Le}^{\mathrm{y}}$ and Le $\mathrm{b}^{\mathrm{b}}$ antigens (Feil et al., 2012). In addition, the structural differences between the $\mathrm{Le}^{\mathrm{y}}$ and $\mathrm{Le}^{\mathrm{b}}$ antigens are minor. These characteristics outline the challenges involved in redesigning LLY ${ }^{l e c}$ in order to increase its specificity for Le ${ }^{y}$. Nevertheless, the results described herein show that, despite the strong structural similarities between Lewis antigens $\mathrm{Le}^{\mathrm{y}}$ and $\mathrm{Le}^{\mathrm{b}}$ and the shallow binding pocket, it is possible to alter the substrate specificity of the LLY lectin domain.

It has previously been demonstrated that $\mathrm{LLY}^{\mathrm{lec}} \mathrm{wt}$ domain binds fucose alone (Farrand etal., 2008; Feil etal., 2012) 
and that the presence of LLYlec enhances the pore-forming activity of LLY on platelets. Fucose is a commonly expressed carbohydrate, terminally decorating cell surface glycoproteins, including CD59 (Rudd et al., 1997; Wheeler et al., 2002), which is also the receptor for LLY (Wickham et al., 2011). This suggests the function of the lectin domain is perhaps as a "capture mechanism," slowing down the passing monomers and increasing the local concentration of LLY in the microenvironment of the cell surface. A localized increase in LLY concentration would facilitate the oligomerization of LLY into prepores, prior to pore formation and consequent cell lysis. In this scenario, weak binding affinities for the fucose moieties, as demonstrated by SPR, would be beneficial as the monomers would need to be readily released for incorporation into assembling prepore oligomers. Further studies are required to confirm this mechanism.

\section{REFERENCES}

Bergelt, S., Frost, S., and Lilie, H. (2009). Listeriolysin $\mathrm{O}$ as cytotoxic component of an immunotoxin. Protein Sci. $18,1210-1220$

CCP4. (1994). Collaborative Computational Project. The CCP4 Suite: programs for protein crystallography. Acta Crystallogr. D Biol. Crystallogr. 50, 760-763.

Clarke, K., Lee, F. T., Brechbiel, M. W., Smyth, F. E., Old, L. J., and Scott, A. M. (2000a). In vivo biodistribution of a humanized anti-Lewis Y monoclonal antibody (hu3S193) in MCF-7 xenografted BALB/c nude mice. Cancer Res. 60, 4804-4811.

Clarke, K., Lee, F. T., Brechbiel, M. W., Smyth, F. E., Old, L. J., and Scott, A. M. (2000b). Therapeutic efficacy of anti-Lewis(y) humanized 35193 radioimmunotherapy in a breast cancer model: enhanced activity when combined with taxol chemotherapy. Clin. Cancer Res. 6, 3621-3628.

Emsley, P., and Cowtan, K. (2004). Coot: model-building tools for molecular graphics. Acta Crystallogr. D Biol. Crystallogr. 60, 2126-2132.

Farrand, S., Hotze, E., Friese, P., Hollingshead, S. K., Smith, D. F., Cummings, R. D., etal. (2008). Characterization of a streptococcal cholesterol-dependent cytolysin with a lewis $y$ and $b$ specific lectin domain. Biochemistry 47, 7097-7107.

Feil, S. C., Lawrence, S., Mulhern, T. D., Holien, J. K., Hotze, E. M., Farrand, S., et al. (2012). Structure of the lectin regulatory domain of the cholesterol-dependent cytolysin lectinolysin reveals the basis for its lewis antigen specificity. Structure 20, 248-258.

Gowda, R. M., Ansari, A. W., and Khan, I. A. (2003). Complete endocardial cushion defect (complete atrioventricular canal) manifested in adult life by Streptococcus mitis endocarditis of the common atrioventricular valve. Int. J. Cardiol. 89, 109-110.

Hall, G. E., and Baddour, L. M. (2002). Apparent failure of endocarditis prophylaxis caused by penicillinresistant Streptococcus mitis. Am. J. Med. Sci. 324, 51-53.

Hotze, E. M., and Tweten, R. K. (2012). Membrane assembly of the cholesterol-dependent cytolysin pore complex. Biochim. Biophys. Acta 1818, 1028-1038.

Huang, I. F., Chiou, C. C., Liu, Y. C., and Hsieh, K. S. (2002). Endocarditis caused by penicillin-resistant Streptococcus mitis in a 12-year-old boy. J. Microbiol. Immunol. Infect. 35, 129-132.

Kennedy, M. J., Jackson, M. A., and Kearns, G. L. (2004). Delayed diagnosis of penicillin-resistant Streptococcus mitis endocarditis following single-dose amoxicillin prophylaxis in a child. Clin. Pediatr. (Phila.) 43, 773-776.

Kitamura, K., Stockert, E., GarinChesa, P., Welt, S., Lloyd, K. O. Armour, K. L., et al. (1994). Specificity analysis of blood group Lewis-y $(\mathrm{Le}(\mathrm{y}))$ antibodies generatedagainst synthetic and natural Le(y) determinants. Proc. Natl. Acad. Sci. U.S.A. 91, 12957-12961.

Kubota, T., Kumagai, A., Ito, H., Furukawa, S., Someya, Y., Takeda, N., et al. (2012). Structural basis for the recognition of Lewis antigens by genogroup I norovirus. J Virol. 86, 11138-11150.

McPhillips, T. M., McPhillips, S. E. Chiu, H. J., Cohen, A. E., Deacon, A. M., Ellis, P. J., et al. (2002). Blu-Ice and the Distributed Control System: software for data acquisition and instrument control at macromolecular crystallography beamlines. J. Synchrotron. Radiat. 9, 401-406.

\section{ACKNOWLEDGMENTS}

This research was partly undertaken on the MX2 beamline at the Australian Synchrotron, Victoria, Australia. We thank Dr. Tom Caradoc-Davies and the other beamline staff for their assistance. We also thank Dr. Jerome Wielens for assistance with X-ray crystallographic data collection. This work was partly carried out in the Australian Cancer Research Foundation Rational Drug Discovery Centre and funding was received from the Victorian Government Operational Infrastructure Support Scheme to St. Vincent's Institute. This research was supported by a Victorian Life Sciences Computation Initiative (VLSCI) grant number VR0021 on its Peak Computing Facility at the University of Melbourne, an initiative of the Victorian Government, Australia. Rodney K. Tweten received support from Grant AI037657 from the National Institutes of Health. Susanne C. Feil was supported by a NHMRC Industry Fellowship. Michael W. Parker is an NHMRC Research Fellow.

Miyake, M., Taki, T., Hitomi, S., and Hakomori, S. (1992). Correlation of expression of $\mathrm{H} / \mathrm{Le}(\mathrm{y}) / \mathrm{Le}(\mathrm{b})$ antigens with survival in patients with carcinoma of the lung. N. Engl. J. Med. 327, 14-18.

Murshudov, G. N., Vagin, A. A., and Dodson, E. J. (1997). Refinement of macromolecular structures by the maximum-likelihood method. Acta Crystallogr. D Biol. Crystallogr. 53, 240-255.

Ohkuni, H., Todome, Y., Okibayashi, F., Watanabe, Y., Ohtani, N., Ishikawa, T., et al. (1997). Purification and partial characterization of a novel human platelet aggregation factor in the extracellular products of Streptococcus mitis, strain Nm-65. FEMS Immunol. Med. Microbiol. 17, 121129.

Otwinowski, Z., and Minor, W. (1997). Processing of X-ray diffraction data collected in the oscillation mode. Methods Enzymol. 276, 307-326.

Phillips, J. C., Braun, R., Wang, W., Gumbart, J., Tajkhorshid, E., Villa, E., et al. (2005). Scalable molecular dynamics with NAMD. J. Comput. Chem. 26, 1781-1802.

Polekhina, G., Giddings, K. S., Tweten, R. K., and Parker, M. W. (2005). Insights into the action of the superfamily of cholesteroldependent cytolysins from studies of intermedilysin. Proc. Natl. Acad. Sci. U.S.A. 102, 600-605.

Rossjohn, J., Feil, S. C., McKinstry, W. J., Tweten, R. K., and Parker, M. W. (1997). Structure of a cholesterolbinding, thiol-activated cytolysin and a model of its membrane form. Cell 89, 685-692.

Rudd, P. M., Morgan, B. P., Wormald, M. R., Harvey, D. J., Van Den Berg, C. W., Davis, S. J., et al. (1997). The glycosylation of the complement regulatory protein, human erythrocyte
CD59. J. Biol. Chem. 272, 72297244.

Sakamoto, J., Furukawa, K., CordonCardo, C., Yin, B. W., Rettig, W. J., Oettgen, H. F., et al. (1986). Expression of Lewisa, Lewisb, $\mathrm{X}$, and $\mathrm{Y}$ blood group antigens in human colonic tumors and normal tissue and in human tumor-derived cell lines. Cancer Res. 46, 1553-1561.

Scott, A. M., Geleick, D., Rubira, M., Clarke, K., Nice, E. C., Smyth, F. E., etal. (2000). Construction, production, and characterization of humanized anti-Lewis Y monoclonal antibody $3 \$ 193$ for targeted immunotherapy of solid tumors. Cancer Res. 60, 3254-3261.

Westwood, J. A., Murray, W. K., Trivett, M., Haynes, N. M., Solomon, B., Mileshkin, L., etal. (2009). The Lewis-Y carbohydrate antigen is expressed by many human tumors and can serve as a target for genetically redirected $\mathrm{T}$ cells despite the presence of soluble antigen in serum. J. Immunother. 32, 292-301.

Westwood, J. A., Smyth, M. J., Teng, M. W., Moeller, M., Trapani, J. A., Scott, A. M., et al. (2005). Adoptive transfer of $\mathrm{T}$ cells modified with a humanized chimeric receptor gene inhibits growth of Lewis-Y-expressing tumors in mice. Proc. Natl. Acad. Sci. U.S.A. 102, 19051-19056.

Wheeler, S. F., Rudd, P. M., Davis, S. J., Dwek, R. A., and Harvey, D. J. (2002). Comparison of the N-linked glycans from soluble and GPI-anchored CD59 expressed in CHO cells. Glycobiology 12, 261-271.

Wickham, S. E., Hotze, E. M., Farrand, A. J., Polekhina, G., Nero, T. L., Tomlinson, S., et al. (2011). Mapping the intermedilysin-human CD59 receptor interface reveals a deep correspondence with the binding site on 
CD59 for complement binding proteins C8 $\alpha$ and C9. J. Biol. Chem. 286, 20952-20962.

Yin, B. W., Finstad, C. L., Kitamura, K., Federici, M. G., Welshinger, M., Kudryashov, V., et al. (1996). Serological and immunochemical analysis of Lewis y (Ley) blood group antigen expression in epithelial ovarian cancer. Int. J. Cancer 65, 406-412.

Yuriev, E., Farrugia, W., Scott, A. M., and Ramsland, P. A. (2005)
Three-dimensional structures of carbohydrate determinants of Lewis system antigens: implications for effective antibody targeting of cancer. Immunol. Cell Biol. 83, 709-717.

Conflict of Interest Statement: The authors declare that the research was conducted in the absence of any commercial or financial relationships that could be construed as a potential conflict of interest.
Received: 05 September 2012; paper pending published: 15 September 2012, accepted: 17 October 2012; published online: 05 November 2012.

Citation: Lawrence SL, Feil SC, Holien JK, Kuiper MJ, Doughty L, Dolezal O, Mulhern TD, Tweten RK and Parker MW (2012) Manipulating the Lewis antigen specificity of the cholesteroldependent cytolysin lectinolysin. Front. Immun. 3:330. doi: 10.3389/fimmu. 2012.00330
This article was submitted to Frontiers in Molecular Innate Immunity, a specialty of Frontiers in Immunology.

Copyright (c) 2012 Lawrence, Feil, Holien, Kuiper, Doughty, Dolezal, Mulhern, Tweten and Parker. This is an open-access article distributed under the terms of the Creative Commons Attribution License, which permits use, distribution and reproduction in other forums, provided the original authors and source are credited and subject to any copyright notices concerning any third-party graphics etc. 